\title{
Les migrations subsahariennes dans les œuvres de création sur TV5 Monde
}

Une programmation entre ruptures et continuités

African Migration in Creation Films on TV5 Monde. Programming between

Ruptures and Continuities

Nathalie Negrel

\section{OpenEdition}

Journals

Édition électronique

URL : http://journals.openedition.org/etudesafricaines/17721

DOI : 10.4000/etudesafricaines. 17721

ISSN : $1777-5353$

Éditeur

Éditions de l'EHESS

Édition imprimée

Date de publication : 30 juin 2014

Pagination : 451-474

ISSN : 0008-0055

\section{Référence électronique}

Nathalie Negrel, «Les migrations subsahariennes dans les œuvres de création sur TV5 Monde »,

Cahiers d'études africaines [En ligne], 213-214 | 2014, mis en ligne le 27 juin 2016, consulté le 01 mai

2019. URL : http://journals.openedition.org/etudesafricaines/17721 ; DOI : 10.4000/

etudesafricaines. 17721

Ce document a été généré automatiquement le 1 mai 2019.

(c) Cahiers d'Études africaines 


\title{
Les migrations subsahariennes dans les œuvres de création sur TV5 Monde
}

\author{
Une programmation entre ruptures et continuités \\ African Migration in Creation Films on TV5 Monde. Programming between \\ Ruptures and Continuities
}

Nathalie Negrel

1 Le traitement des migrations est un réel défi à relever pour les journalistes. Ce phénomène complexe, mobilisant des thèmes nationaux comme internationaux, touchant à des sujets sociaux, économiques, politiques et culturels perturbe ces frontières classiques que les rédactions s'imposent pour rendre compte d'un sujet. Jean-Paul Marthoz, journaliste et chercheur, parlera des migrations comme d'un sujet " glocal $»^{1}$, au croisement entre le local et le global, nécessitant de revenir sur les partitions d'usage (ici par exemple entre information locale, nationale ou internationale) au sein des rédactions médiatiques.

2 Notre propos sera ici, dans une perspective communicationnelle, d'interroger la place des récits de migrations dans les œuvres de création ${ }^{2}$ programmées par la chaîne internationale francophone TV5 Monde. Alors que Jean-Paul Marthoz préconise de multiplier les points de vue sur un sujet qui présente autant de facettes et d'enjeux, la présence de ces discours non journalistiques au sein d'une programmation éclectique soulève des enjeux bien particuliers. Nous partirons alors des propositions de Michèle Gellereau (1998 : 97) dans son article « Dispositif télévisuel et médiations : une étude de documentaires et reportages consacrés aux gens ordinaires ", où elle montre " comment un même projet de refus des stéréotypes attachés aux images médiatiques de personnes dites "en difficulté" ou "exclues" conduit des auteurs de documentaires ou de reportages audiovisuels à créer un dispositif de médiations qui vise à rendre la complexité de la réalité ». 
3 Nous reprendrons la définition de la médiation proposée qui trouve son origine dans un contexte audiovisuel qui connaît une "rationalisation et réduction du nombre de discours et de références " et où il importerait de "préserver les distances entre les expériences, les vocabulaires, les symboles» (Wolton 1997: 166). Par conséquent, la médiation «n'est ni réunion ni intercession, laquelle suppose une vérité donnée et une hiérarchie, elle est avant tout reconnaissance des différences, reconnaissance qu'il y a des oppositions entre les représentations et les symboles des uns et des autres; elle est aussi bien sûr, la base de cette reconnaissance, travail de relation, de compréhension entre des sujets, expression de l'épaisseur et des contradictions de la réalité. Dans le rapport au monde réel, l'idée de médiation nie celle de la transparence, de l'évidence, l'illusion d'une compréhension immédiate et rappelle que la connaissance se construit dans une mise en commun du monde. Dans les relations entre groupes et personnes, la médiation permet à deux sujets, deux mondes, deux communautés de se comprendre, de développer des relations mais sans fusionner " (Gellereau 1998 : 97).

4 Tout en nous focalisant sur cet ensemble d'œuvres de création ${ }^{3}$, nous élargirons cette réflexion à la logique de programmation de la chaine internationale TV5 Monde, incluant alors l'ensemble des documents audiovisuels qui constituent notre corpus global (reportages, émissions de plateaux, ainsi que documentaires et films de fiction). L'approche des chaînes de télévision comme appartenant à la sphère publique médiaculturelle 4 nécessite en effet de les appréhender au travers de la globalité de leur programmation, sans séparer les programmes d'information d'un côté, et les programmes culturels de l'autre. Au sein du paradigme des médiacultures, la sphère publique «loin d'être un espace rationnel, [...] est pensée comme le lieu d'un intense conflit de définition entre acteurs inscrits dans des rapports sociaux et des mouvements culturels divergents » (Macé \& Maigret 2005: 49). Ce "conflit de définition» constitue tout l'enjeu des représentations médiatiques : « nommer, représenter, c'est à la fois faire exister et définir un cadre interprétatif plus ou moins hégémonique ou subversif » (ibid.).

5 Ainsi, à travers la comparaison des genres audiovisuels ${ }^{5}$ qui appartiennent à notre corpus, nous tenterons de comprendre les enjeux spécifiques de la diffusion de ces œuvres de création sur la chaîne TV5 Monde. Pour cela, nous commencerons par présenter notre méthodologie et notre corpus. Puis nous nous intéresserons à l'instance de diffusion des films que nous étudions, en nous focalisant sur les relations qu'entretient TV5 Monde avec le continent africain. Nous verrons que les dispositifs d'énonciation mis en place dans les différents portraits remettent le migrant au centre de l'attention, le positionnant comme un sujet social. Les cadrages choisis diffèrent alors nettement, posant la question du « décentrage/recentrage » des points de vue. Enfin, nous terminerons en revenant sur les conditions de production et de diffusion de ces films en examinant les enjeux de la circulation internationale de ces discours.

\section{Méthodologie et corpus}

6 Notre analyse prendra pour cadre une méthodologie sémio-pragmatique, interrogeant les discours émis, mais également les conditions de production et de diffusion de ces discours.

7 Notre corpus global est composé de l'ensemble des documents traitant des migrations subsahariennes dans la programmation de la chaîne internationale TV5 Monde ${ }^{6}$. Trente 
documents, dont huit œuvres de création, ont été rassemblés sur la base de l'indexation de l'intégralité de la programmation des deux chaînes par les documentalistes de l'INA'. Le travail des documentalistes consiste donc à visionner "les émissions récupérées auprès des diffuseurs et les interpréter sous forme de mots, dans des notices documentaires ${ }^{8}$. La rédaction d'une notice passe par deux étapes: le «catalogage " (avec des données comme le titre, la date de diffusion, le nom du réalisateur, etc., ainsi que la définition du genre du document) et l'« indexation » avec un résumé en texte libre et l'attribution de mots-clés issus du thésaurus9. Bruno Bachimont (Lespinasse, Habert \& Bachimont 2000 : 3) relève alors les principaux enjeux liés à cette indexation : «La part d'interprétation dans les notices, son influence sur la recherche documentaire; le peu de contrôle sur la terminologie [...]: le résumé est de rédaction entièrement libre; [...] et l'usage d'un vocabulaire normé, ici un thésaurus, dont le volume, à savoir plus de 10000 noms communs, dépasse les capacités de mémoire humaines ; le volume de documents à traiter, que ce soit au niveau de l'indexation ou lors de la recherche des documents. »

Le critère de recherche que nous avons sélectionné a été la présence de la racine « migr » ${ }^{10}$ dans le résumé ou dans les descripteurs, la sélection géographique ayant ensuite été réalisée par nos soins, document par document (nous ne nous intéressons qu'aux migrations subsahariennes). Si « la part d'interprétation évoquée justifie l'impossibilité de systématicité et de cohérence strictes $»^{11}$, il faut noter que les documentalistes de l'INA travaillent avec un guide explicitant règles et usages de l'indexation, ayant justement pour objectif de renforcer la pertinence des notices rédigées: Manuel d'indexation. Le traitement documentaire des programmes de radio et de télévision à l'Inathèque. Si le choix de l'entrée par cette famille de mot, ainsi que l'entrée par la mise en texte reste arbitraire, ils garantissent néanmoins la cohérence et l'intérêt de notre corpus.

Notre étude s'appuie sur l'analyse des huit films que nous définirons comme des œuvres de création audiovisuelles. Cette appellation, issue du monde professionnel, a pour nous l'avantage d'expliciter la distinction que nous faisons entre ces films et les autres documents que nous étudions par ailleurs et qui sont le produit d'un discours journalistique. Nous séparons donc notre corpus global en deux sous-parties, composées d'une part des œuvres de création (films documentaires ainsi que films de fiction, courtsmétrages ou moyens-métrages) et, d'autre part, de discours journalistiques (reportages, magazines ou émissions de plateau).

cous restreint que nous nous proposons d'étudier ici tire une grande part de sa valeur grâce à sa singularité vis-à-vis des discours journalistiques. Notre approche sera donc comparative. Tout en constituant une méthode d'analyse, la comparaison représente plus qu'un outil. Elle «doit ainsi être conçue comme une démarche, un état d'esprit destiné à déplacer le regard du chercheur. Comparer, c'est en effet non seulement accepter de se décentrer, mais également rendre plus exigeants la formulation d'hypothèses et le travail de théorisation » (Vigour $2005: 18$ ).

11 Précisons toutefois que l'intérêt de l'analyse de ces œuvres de création ne réside pas seulement dans la comparaison et possède également des enjeux qui leur sont inhérents. Puisque ces documents ne proviennent pas de journalistes chargés de rendre compte de l'actualité, nous nous demanderons qui sont les personnes ayant pris en charge ces discours? De quelles natures sont-ils ? Dans quels cadres sont-ils diffusés sur la chaîne et quels enjeux sont soulevés par leur diffusion dans un espace médiatique international ? 

fictions: Vacances au Sénégal (Christophe 2005); Vivre de sable (Diakite 2007); Expectations (Haroun 2008); Un ami est parti (Kifouani 2008); Aminata rencontre Aminata (Lion 2008); Atterrissage. Le message de Yaguine et Fodé (Nice 2007) ; Le cri de la mer (Thiam 2008); Afrique Années 60 (Wouassi 2003).

L'objectif de notre analyse ne sera donc pas d'étudier les choix esthétiques présents dans ces films, mais bien d'analyser les traits communs ou dissemblables des portraits des migrants subsahariens et d'en relever les enjeux, notamment à travers la comparaison avec certains des résultats de l'analyse du corpus global qui est le nôtre. Deux outils ont été mis en place : d'un côté une grille d'analyse systématique qui a permis de relever les mêmes données pour chacun de nos films ${ }^{12}$, de l'autre côté, des entretiens avec les réalisateurs et des salariés des structures participant à la production et diffusion des programmes ${ }^{13}$.

\section{TV5 Monde, « une tribune pour l'Afrique ${ }^{14} ?$}

Née à Paris en 1984, sous l'impulsion du ministère des Affaires étrangères français du gouvernement de Pierre Mauroy, TV5 Monde est le fruit de la coopération entre le secteur audiovisuel public français et les chaînes publiques suisse et belge, ainsi que le Consortium de télévisions publiques québécoises et canadiennes (стQC). Chaîne multilatérale ${ }^{15}$, TV5 Monde fonctionne avec une grille de programmes qui se compose à la fois de la rediffusion de programmes des chaînes partenaires ( $45 \%$ ), de la diffusion de programmes produits directement par la chaîne $(19 \%)$ et d'achats ou préachats de programmes $(36 \%)^{16}$.

S'adressant tout particulièrement aux expatriés français, à la communauté francophone, ou encore aux francophiles, la chaîne diffuse huit signaux régionalisés: l'Europe francophone (canal FBS), l'Europe non francophone, l'Afrique, l'Asie, le Pacifique, le Maghreb-Orient, l'Amérique latine et les États-Unis. Une chaîne spécifique destinée au territoire canadien est dirigée par TV5-Québec Canada, structure indépendante basée à Montréal.

16 TV5 Monde est particulièrement bien implantée en Afrique de l'Ouest («plus de 2,6 millions de téléspectateurs quotidiens sur 10 villes, soit près d'un quart de la population de 15 ans et plus ») $)^{17}$, avec qui elle entretient une relation privilégiée que ce soit à travers sa distribution, la structure de son site Internet (avec un portail spécifiquement consacré à l'Afrique), la présence de vingt maisons TV5 Monde sur le continent, ou encore la naissance de sa WebTV Afrique en 2010.

17 Force est de constater alors que le continent africain est le seul qui possède sur la chaine des programmes qui lui sont spécifiquement dédiés. Ainsi, tous les jours, sur tous les signaux de la chaîne, le «Journal international » de la rédaction est suivi du "Journal Afrique ». De la même façon, depuis 2008, avec le nouveau plan stratégique mis en place par l'ancienne directrice générale de TV5 Monde, Marie-Christine Saragosse ${ }^{18}$, les nombreuses émissions consacrées à l'actualité, à la culture africaine, à l'économie ne sont plus seulement diffusées sur le canal africain mais le sont également sur le canal FBS et le canal Europe, ainsi que, pour certaines, sur le canal Amérique latine. L'ancienne directrice a annoncé en effet souhaiter « rétablir la place de l'Afrique sur tous les signaux mondiaux $»^{19}$. 


\section{Dispositifs d'énonciation, le migrant comme sujet social}

18 «L'énonciation n'est pas cette scène illusoire où viendraient se dire des contenus élaborés ailleurs, mais un dispositif qui est partie prenante dans la construction d'un sens et des sujets qui s'y reconnaissent" (Maingueneau 1987: 35). Les dispositifs bien particuliers sur lesquels nous allons revenir ici participent, selon nous, non seulement de la construction du sens des différents films, mais également de la manière d'aborder les personnes qui apparaissent à l'écran ou dont il est question. Nous prendrons le temps ici de détailler différents dispositifs afin d'illustrer nos propos et de pouvoir rendre compte des propositions qui nous sont faites par les huit réalisateurs.

19 L'analyse des documents journalistiques montre bien comment la parole des migrants est très peu présente, supplantée par celles des journalistes, des hommes politiques et des experts. Les migrants sont ainsi davantage objets de discours qu'énonciateurs; ils apparaissent à l'écran pendant que d'autres décrivent, analysent, dénoncent, débattent sur les questions migratoires. Les migrants sont représentés plutôt comme des objets muets ; ils apparaissent à l'écran sans que l'on n'entende leur voix. Dans la continuité de la figure du migrant objet de discours, le migrant à l'image se situe de son côté au service du discours des journalistes. Différents cas de figure apparaissent alors : les images sont diffusées en illustration des propos tenus, en même temps qu'un débat a lieu sur le plateau ; les images d'un reportage viennent initier ou interrompre un débat sur plateau pour l'introduire, l'illustrer ou annoncer une nouvelle thématique; les images d'un reportage où le fil conducteur est constitué par le discours du journaliste, les images venant alors en appui du discours.

20 Les quelques prises de parole de la part des migrants sont la plupart du temps extrêmement courtes, et ne viennent qu'en appui du discours du journaliste ou de l'expert. Nous rejoignons alors Guy Lochard (1995: 163) quand il montre que les exigences médiatiques peuvent contraindre les prises de parole des "personnes ordinaires ", à tel point qu'elles deviennent « un objet de la représentation télévisuelle ».

21 Les œuvres de création qui nous intéressent ici se distinguent nettement dans les différents dispositifs mis en place pour mettre en valeur les paroles des migrants ainsi que leurs émotions. Dans les deux courts-métrages de fiction, les migrants, même s'ils n'ont pas le même statut et le même parcours, s'expriment davantage à travers leur corps qu'à travers des mots. Dans Afrique Années 6020 (Wouassi 2003), deux jeunes gens se croisent à travers la musique de Manu Dibango et la danse. Les deux voix off qui se font entendre par intermittence permettent alors l'évocation de sensations, couleurs, odeurs, chaleur à travers des éléments charnels comme la nourriture, la danse, la sensualité. «J'ai 7 ans Manu, le soleil cogne mon crâne et mes pieds nus, rouges de la poussière de Yaoundé, rouges de ma terre. Vole vers ma maison, ma maman m'attend avec un fufu brûlant. Oui Manu, je fais mon éducation sur ta musique et dans les bals de la brique. »

22 C'est donc un rapport extrêmement sensible qui se fait jour dans le rappel du pays d'origine à travers un dialogue entre les deux protagonistes, la musique qui s'incarne dans le prénom de son auteur "Manu », à qui s'adressent les deux jeunes, et leur corps sur lesquels la caméra s'attarde. Le choix du musicien camerounais dont le parcours personnel et musical est marqué par les allers-retours entre les continents africain, 
européen et américain est alors symptomatique, tout comme le rôle moteur joué par sa musique dans le scénario qui rend prégnante l'approche sensuelle de la partition entre pays d'accueil et pays d'origine.

Dans Expectations ${ }^{21}$ (Haroun 2008), la première partie qui se déroule dans le désert se construit autour des corps en souffrance, victimes de la soif, de la chaleur et de l'épuisement ; la seconde partie, qui décrit le retour au village, révèle la folie de celui qui est privé de parole, dont le corps ne répond plus. Les mots qui se font alors entendre avec violence sont ceux de l'entourage qui ne peut accepter son retour et le pousse à repartir.

« Regarde moi ces cheveux. C'est trop long. Coupe-les. Tout le monde dit que tu es devenu fou. Mais en réalité, personne ne croit en ta folie. Tu imagines toutes ces dettes que tu as contractées pour partir. C'est la deuxième fois, et tu as encore raté ton voyage. Les dettes s'accumulent et les créanciers s'impatientent. »

Les discours normatifs des proches à propos de l'apparence physique du migrant en échec reflètent le refus de la communauté de cet échec et de la présence parmi eux de cet homme devenu indésirable.

Incarnée, charnelle, l'expérience migratoire est ainsi rendue présentée à travers des aspects concrets et non pas seulement à travers des raisonnements abstraits et conceptuels.

Face à l'absence de ceux qui sont morts sur le trajet, les paroles des proches sont aussi des discours médiateurs pour comprendre, témoigner, se battre. Le documentaire Atterrissage. Le message de Yaguine et Fodé22 (Nice 2007) montre ainsi la lecture sur scène par les comédiens de la lettre des deux jeunes adolescents disparus :

« Messieurs les membres et responsables d'Europe, c'est à votre solidarité et à votre gentillesse que nous vous appelons au secours en Afrique. Aidez-nous. Nous souffrons énormément en Afrique. Aidez-nous, nous avons des problèmes et quelques manques de droits de l'enfant. »

Si ces mots sont ainsi à nouveau rendus audibles, le documentaire filme également les rencontres qui sont organisées avec des collégiens, des lycéens et des orphelins accueillis par la Croix-Rouge au Bénin. À travers les nombreux moments où les jeunes s'expriment devant la caméra, c'est l'occasion pour la réalisatrice de donner la parole à la génération de Yaguine et Fodé. Un dialogue apparaît alors pour le téléspectateur entre les interventions des jeunes, de la ministre de l'Enseignement, des comédiens « issus de la diaspora ", comme ils se définissent eux-mêmes, des professeurs qui évoquent également le départ de la plupart des professeurs en Europe.

Le documentaire Un ami est parti23 (Kifouani 2008) témoigne de son côté d'un double rapport à la parole. Si le film est né de la souffrance d'un départ sans explications, il se fait alors lui-même lettre, adressée à celui qui est parti, et évocation de la relation si chère qui s'était établie entre eux. Le film est ainsi l'occasion pour le réalisateur d'interroger sa propre expérience migratoire, son départ du Congo pour venir étudier au Sénégal. Cette expérience passe en particulier par les mots, les mots pour nommer une végétation inconnue, mais aussi les mots avec lesquels les Sénégalais le désignent.

«C'est avec toi que j'ai osé parler pour la première fois de ma différence. Tu m'as appris que les Sénégalais m'appellent Niak parce que je viens d'Afrique centrale. Tu m'as écrit ce mot sur un bout de papier. »

La mise en abyme de la migration Sud-Sud à l'intérieur de cette «lettre " pour un ami parti au Nord permet ainsi de faire naître une analyse inédite sur la xénophobie en Afrique, illustrée ici dans les relations entre Sénégalais et Congolais. 

documentaires s'inscrivent dans la série «Femmes battantes » de l'émission «Reflets Sud $»^{24}$ et mettent en scène la migration à travers des personnages féminins. Avec Vivre de sable ${ }^{25}$ (Diakite 2007), le ton est donné avant même le générique où une femme interpelle la caméra avec virulence : "Il faut que cette souffrance et cette misère cessent. » Aucun commentaire ne viendra s'ajouter aux voix des femmes sous-titrées en français. La réalisatrice s'efface derrière les mots des villageoises. qui tente de se faire entendre et de sensibiliser l'ensemble de sa communauté aux dangers du départ en pirogue. La question du discours est donc au centre du documentaire, dès le titre, puis dans le déploiement des activités de l'association (émissions radios, palabres, etc.) qui semblent compenser le mutisme des hommes, en l'occurrence le mari : «Mon mari est très affecté, il ne peut pas parler ", et les survivants : "Ce que j'ai vécu est tellement dur que j'ai du mal à trouver les mots. J'ai du mal à croire que je suis en train de vous parler.»Cette question du témoignage, de la prise de parole du migrant ou de ses proches donne alors une nouvelle dimension à ces récits qui donnent la parole à ceux que l'on n'entend pas dans le flux médiatique. Les deux derniers exemples qui mettent en avant des portraits de «femmes battantes » offrent alors un contre-point supplémentaire en amenant sur le devant de la scène des personnages féminins.

Enfin, les deux documentaires qui traitent du retour dans le pays d'origine permettent d'explorer le point de vue du réalisateur qui effectue lui aussi le voyage et dialogue à cette occasion avec ses propres représentations. Dans Vacances au Sénégal ${ }^{27}$ (Christophe 2005), le réalisateur qui avait dans un film précédent (ibid. 2003) servi de médiateur entre la famille sénégalaise de son ami et ce dernier, sans-papiers en Italie, filme ici les retrouvailles, les joies et les incompréhensions. De ces rencontres naîtront de nombreuses interrogations pour le réalisateur qui découvre certaines facettes de la vie de son ami, en particulier sa relation avec son épouse, avec laquelle il s'est marié à distance. Le regard subjectif du réalisateur, un français « adopté » par cette famille sénégalaise, et le dialogue permanent avec son ami permettent d'accéder à une description originale et circonstanciée de la société sénégalaise. Les rapports familiaux, amoureux, la relation à la distance, à l'argent, aux générations sont ainsi décrits, parfois interrogés, avec tendresse ou scepticisme.

Dans Aminata rencontre Aminata ${ }^{28}$ (Lion 2008), le réalisateur met en scène le dialogue difficile entre Aminata, fille française de migrants, et les jeunes Sénégalais qu'elle essaie de dissuader de partir :

«- En tout cas, je sais pas pour la génération de ton papa, mais la génération actuelle, ceux qui font l'aventure, ils vont pas en Europe pour s'installer, la plupart des cas, ils vont en Europe pour quelques temps...

- Combien d'années ? Cinq ans?!

- L'objectif, c'est d'aller chercher de l'argent et de revenir au Sénégal pour faire sa vie. Actuellement, c'est ce que font les jeunes...

- Après, je ne peux pas vous dire n'y allez pas. Ben oui, de toute façon, si franchement vous y arrivez, j'aimerais bien vous voir dans cinq ans pour voir quelle fortune vous aurez pour revenir.

- Le problème, c'est qu'on peut pas voir de la même façon que toi. Parce que toi, tu $\mathrm{y}$ vis. Tu vis en France...

- C'est pour ça que cette discussion, elle est intéressante, mais quelque part, elle mène nulle part parce que dans tous les cas, ce que je vous dis. Sur $100 \%$ de ce que 
je vous dis, vous allez pas prendre les $100 \%$, vous allez prendre, quoi, les $20 \%$ qui

vous arrangent et vous laissez le reste. » vue politique et/ou économique. Ce que nous qualifierons de «regard gestionnaire» permet essentiellement de développer les différents aspects du «problème de l'immigration » en se concentrant sur la gestion des flux migratoires, la gestion des frontières, les politiques migratoires avec l'évolution des législations françaises ou européennes. La diffusion d'un reportage sur cette question et/ou son traitement dans un débat sont donc directement liés à l'agenda politique, amenant un point de vue administratif, législatif ou comptable ${ }^{29}$. 
38 En outre, la médiatisation des tentatives de passage à la frontière, qu'il s'agisse de la frontière terrestre (au niveau des enceintes espagnoles de Ceuta et Melilla) ou des frontières maritimes (mer Méditerranée ou océan Atlantique), est majoritairement ramenée au cadrage politique et institutionnel. Il se traduit par la focalisation sur l'espace méditerranéen, qui se retrouve au centre de toutes les attentions avec la question des accords bilatéraux entre pays européens et africains, de l'externalisation des frontières européennes, avec parfois une excursion vers la question du non-respect des droits de l'homme aux frontières que les pays maghrébins prennent en charge.

Marginal, un troisième angle apparaît cependant de manière épisodique, celui de l'actualité culturelle. En dehors de la programmation des œuvres de création, c'est l'occasion pour la chaîne de présenter des auteurs, des œuvres ayant pour sujet les migrations subsahariennes et apportant un autre regard sur la question. Les intellectuels africains ou d'origine africaine invités sur les plateaux deviennent les porte-voix des migrants, racontant des trajectoires parfois personnelles, parfois fictionnelles, redessinant alors à travers leur présence sur le plateau une figure du migrant, symbole fantasmé de l'intégration achevée.

Dans les œuvres de création, les angles d'attaque qui s'imposent ne sont plus ceux qui adoptent les points de vue politiques de l'Union européenne ou de la France, mais deviennent personnel, familial ou encore communautaire. Les enjeux ne sont donc plus les mêmes et ce qui est traité comme une expérience plutôt que comme un phénomène, dévoile alors de nouvelles facettes.

41 D'un point de vue géographique tout d'abord, ce ne sont pas de nouveaux pays qui apparaissent, mais de nouvelles trajectoires. Deux films interrogent le trajet inverse, celui qui part du pays d'émigration vers le pays d'origine (de l'Italie vers le Sénégal pour Yelli ; de la France vers le Sénégal pour Aminata). En outre, les films Un ami est parti (Kifouani 2008) et Vivre de sable (Diakite 2007) font état des migrations internes (du village vers la ville au Niger) et Sud-Sud (du Congo vers le Sénégal) :

«L'ami dont je parle dans le film, c'était justement le premier qui m'a dit que ce qui se faisait en Afrique centrale ne se faisait pas ici. Je prends un exemple : chez moi, en fait au Congo, c'est très mal vu d'aller manger dans d'autres familles. Mais en Afrique de l'Ouest, c'est le contraire. Quand on invite à manger et que tu déclines l'offre, c'est très mal vu. Donc il m'a fallu des clés, des codes pour comprendre dans quelle société je me retrouvais. Et ces clés, c'est cet ami qui me les a données. Donc à partir de là, je me suis dit qu'il était intéressant de parler de cette immigration Sud-Sud. Parce que généralement, ce qui défraie l'actualité c'est l'immigration Nord-Sud. Parce que moi, quand je suis en Europe, on me regarde et on me dit que finalement, je suis un Africain, je suis un Noir. Alors qu'à l'intérieur de l'Afrique, il y a des différences, il y a des contradictions. Je voulais vraiment traiter de cet aspectlà $»^{30}$.

Alors que ces migrations sont statistiquement majoritaires, elles n'intéressent que très peu les rédactions des médias internationaux ${ }^{31}$. Le film de Delphe Kifouani (2008) ne développe pas seulement cet aspect géographique mais propose une analyse sociale des différences existant entre les différents pays africains et les implications qu'elles peuvent avoir pour les populations.

Autre particularité qui apparaît nettement, ces films interrogent pour la plupart les raisons du départ alors que dans les discours journalistiques, l'attention reste plutôt focalisée sur le trajet ou l'arrivée. Si le point de vue du départ est parfois abordé, c'est à travers la création de centres d'information, gérés par les pays européens dans l'objectif 
de canaliser les départs vers l'Union européenne ${ }^{32}$. Dans les œuvres de création, les raisons du départ sont alors expliquées, évoquées, questionnées, inscrivant les départs dans une réelle trajectoire, dans un récit de vie ne débutant pas le jour du départ.

Quand il est question du trajet vers l'Europe et de ces aventures qui se terminent de façon tragique, c'est le point de vue des proches qui est donné et non celui des autorités. Ainsi dans Le cri de la mer, Aïcha Thiam (2008) travaille sur la question de la prise en charge du discours :

« Moi j'avais envie de faire un film d'un point de vue sénégalais. Parce qu'on a fait tellement de films où on critique l'Occident... Je voulais expliquer du point de vue sénégalais pourquoi ces jeunes partent. Eh bien, c'est le gouvernement sénégalais qui vend ces licences de pêche. C'est l'urbanisation galopante, il n'y a plus d'espaces pour cultiver. La terre ne nourrit plus son homme, la mer ne donne plus de poissons. L'État n'est pas là pour créer des activités génératrices de revenus pour ces jeunes. Il y a le chômage $\aleph^{33}$.

De son côté, la réalisatrice du film Atterrissage. Le message de Yaguine et Fodé (Nice 2007) met en avant les parties de la pièce qui mettent en scène la mère d'un des deux jeunes garçons. Enfin, dans Expectations, Mahamat-Saleh Haroun (2008) ne s'intéresse pas à ceux qui meurent dans le désert, mais au devenir de ceux qui survivent et rentrent chez eux tout en ayant échoué. Alors que dans la fiction, le rescapé ne prononce pas un mot à partir du retour dans son village, il se verra assailli de questions et reproches de la part de tous ses proches. Le spectateur qui a pu se rendre compte du courage et de la persévérance de Moussa dans le désert ne peut que souffrir avec lui de l'injustice des reproches qui lui sont faits. Dans l'absence de réponse de la part du protagoniste, le spectateur ne peut que répondre lui-même aux allégations portées tout en reconnaissant la part d'angoisse et d'incompréhension qui explique la dureté des réactions de sa famille. Les réalisateurs opèrent donc un retour vers le pays d'origine, revenant sur les répercussions d'un échec pour les proches et la communauté de celui qui n'a pas atteint sa destination. Les différents dispositifs de mise en scène, faisant intervenir avec force les émotions tout en permettant aux spectateurs de rester en retrait, mettent en valeur cette dimension originale et ces nouveaux points de vue.

Notons tout de même le peu de place fait à l'espace européen. Les œuvres de création rejoignent en cela les discours journalistiques des deux chaînes internationales étudiées, qui se concentrent sur les migrants en chemin. TV5 Monde diffuse toutefois depuis 2008 une série intitulée «Les immigrés » et qui filme les aventures d'une famille africaine installée à Paris. Commandée par la chaîne et réalisée par le Congolais James Lukezo, elle n'est diffusée que sur le canal africain, ainsi que sur la webTV, d'où son absence de notre étude.

De l'expression de ces nouveaux points de vue émergent alors de nouvelles modalités discursives. De nouvelles manières de nommer la migration, présentée comme une expérience et non plus comme un phénomène, confortent l'opposition que nous décrivions précédemment entre un point de vue gestionnaire et un point de vue singulier. Première conséquence, les discours ne sont pas toujours militants, comme ils peuvent l'être parfois dans d'autres productions. Rappelons qu'Édouard Mills-Affif (2002) avait montré dans sa thèse Filmer les immigrés, que les films documentaires et les reportages diffusés dans les années 1960 à la télévision française étaient des films engagés, très politiques, où la défense de l'immigré servait le discours des réalisateurs communistes de l'époque (comme Jacques Krier) ou des réalisateurs chrétiens. Deux documentaires de notre corpus nous permettent de nuancer ce constat. En effet, les deux réalisatrices du Cri 
de la mer et d'Atterrissage. Le message de Yaguine et Fodé revendiquent un engagement militant dans leur film :

«Mais pourquoi, moi je me demande aussi, pourquoi mon fils qui a quinze ans, il suffit qu'il mette son derrière dans un bel avion, et il peut aller à peu près où il veut sur la planète. Il aura un visa facilement, il a des parents qui paient son billet d'avion. Pourquoi mon fils a le droit au voyage et pourquoi Yaguine et Fodé n'ont pas... Pourquoi les millions de Yaguine et Fodé n'ont pas droit au voyage? Donc voilà c'est ça la logique militante. C'est un cri de révolte contre l'injustice $»^{34}$.

De son côté, Aïcha Thiam nous expliquait :

«Moi je dis toujours "prendre la caméra et filmer, c'est un acte politique". Parce qu'on pose son regard sur quelque chose, un discours. Pour moi, il n'y a pas plus politique que ça [...]. Moi, quand je faisais Le cri de la mer, j'avais peur que le film soit censuré ! Parce que j'ai décidé de critiquer l'État sénégalais, de critiquer en premier lieu nos dirigeants. De critiquer l'État sénégalais qui vend ces licences de pêche. De critiquer l'État sénégalais de laisser ces jeunes en rade, qui n'ont rien du tout. Y'a pas d'activités génératrices de revenus! Y'a pas de programmes d'insertion professionnelle! Y'a pas de fonds qui financent ces jeunes dans ce qu'ils ont envie de faire! Y'a rien pour eux! J'avais peur moi, je me disais "Olala ce film va pas passer !" Mais le film est passé ! »".

Il est alors intéressant de constater des similitudes dans les parcours de ces deux réalisatrices, journalistes à plein temps, et qui développent en parallèle des projets plus personnels à travers la réalisation de documentaires. Quand les enjeux politiques ou diplomatiques des migrations sont laissés de côté, ce sont des sensations, des émotions, des sentiments qui sont exprimés avec les mots, des mots qui expliquent également la faim, la pauvreté, la souffrance. Des termes, que les journalistes n'évoquent pas, affleurent dans les récits, rendant compte des expressions qui circulent : la migration est une "aventure ", le migrant un "aventurier ", celui qui revient au pays en se faisant valoir un «fanfaron ». Les Sénégalais ont leurs slogans «Mbeuk Mi» («l'aventure par la mer ») et « Barça ou Barsakh» (« Barcelone ou la mort »).

Les auteurs travaillent également à la mise en scène des migrations à travers de nouveaux dispositifs comme l'exprime Delphe Kifouani :

"Je voulais trouver une façon originale de filmer l'absence. Filmer le drame, filmer l'immigration, sans recourir à ce qui se fait habituellement. Montrer les photos de celui qui n'est plus. Donc je m'étais dit, dans tous les films que j'ai regardés sur l'immigration clandestine, sur les pirogues en partance pour l'Espagne... Finalement, je trouvais que ces images étaient un peu rabâchées et que je voulais trouver, en tout cas, une autre façon astucieuse de filmer l'absence. Voilà, alors je filme où on a été ensemble avec cet ami. Et il y a une voix off très personnelle, qui rend compte de ce voyage que j'ai fait moi-même vers l'inconnu, et ce voyage qu'il a fait lui aussi, sans donner de suite $»^{36}$.

Plusieurs réalisateurs affirment donc concrètement leur volonté de produire un nouveau discours, original, parfois clairement assumé comme politique, sur les migrations, qui viennent contrecarrer ou tout au moins renouveler les représentations offrant un point de vue sur la question. Il est donc également question de l'émergence d'une diffusion audiovisuelle au niveau international d'un imaginaire africain de la migration. Les logiques spatiales apparaissent ici primordiales. Elles reflètent tout à la fois la dimension intrinsèquement géographique des migrations qui se retrouve redéfinie ici par ces œuvres de création. Alors que sur le plan international, les flux audiovisuels perpétuent la «dialectique du centre et de la périphérie» (Kane 2004: 44), ce pan de la programmation de TV5 Monde remet au centre des espaces marginaux dans les 
programmes audiovisuels occidentaux. Le Bénin, le Sénégal, le Niger redeviennent des lieux d'où l'on peut penser et dire la migration.

Afin d'interroger la notion de décentrage, déterminante pour comprendre ces processus médiatiques, deux niveaux d'analyse doivent être distingués : celui de la chaîne qui opère un décentrage dans le paysage audiovisuel global en programmant ces œuvres, et celui des films que nous étudions et qui effectuent de leur côté un recentrage ${ }^{37}$ du regard sur la question des migrations. Distinguons notamment les films des deux réalisateurs français qui filment le retour de deux personnes dans leurs familles respectives au Sénégal pour lesquels nous pouvons parler de décentrage du regard. Le mélange de tous ces points de vue permet de replacer les migrations dont il est question dans un contexte glocalisé, alliant d'une part le local et le global, mais permettant également de distinguer différentes définitions du local ${ }^{38}$.

\section{TV5 Monde, la « plus grande salle de cinéma africain du monde »?}

53 La diffusion de nouveaux discours permet d'interroger la circulation des récits des migrations et le statut qu'ils peuvent prendre selon les contextes dans lesquels ils se situent. Il est alors intéressant de noter que cinq de ces huit œuvres ont été diffusées lors de l'émission «Reflets Sud $\aleph^{39}$, une collection coproduite par le CIRTEF (Conseil international des radios et télévisions d'expression francophone), présidé depuis 2011 par François Guilbeau, directeur de France 3 et la RTBF, chaîne de télévision belge.

«Reflets Sud» est décrite par ses producteurs comme le «magazine de la francophonie plurielle», "miroir authentique d'un Sud trop souvent associé à des images de désolation, de misère et de souffrance ». Au travers des reportages diffusés sur tous les canaux de TV5 Monde, cette émission hebdomadaire a pour objectif de présenter dans des programmations au Nord «le Sud qui travaille, qui lutte pour s'en sortir, un Sud aux richesses culturelles et humaines incroyables et inouïes $»^{40}$. Lors de l'émission fêtant ses vingt ans, la présentatrice revenait sur sa philosophie : «Seul mot d'ordre : éviter les clichés » et annonçait le florilège des extraits de la façon suivante: "Ensemble nous allons marcher sur les traces d'hommes et de femmes qui ont fait les bonheurs de "Reflets Sud". Leurs récits, petits ou grands, incarnent leurs visions du monde qu'ils nous ont proposées $»^{41}$. L'instance de diffusion annonce donc clairement s'opposer à certains discours unidimensionnels et également passer par des récits singuliers et personnels.

Les œuvres diffusées sur TV5 Monde dans ce cadre de coopération ne sont pas directement le résultat d'un choix de programmation de la part de la chaîne. Leur diffusion est toutefois issue de la réflexion concertée que mène le CIRTEF sur la circulation des discours et des points de vue venus du Sud. Mafarma Sanogo, membre du secrétariat général du CRTEF affirme ainsi que l'objectif de sa structure est de promouvoir un regard audiovisuel africain, afin de proposer aux téléspectateurs différents points de vue sur les réalités africaines et de leur permettre d'exercer leur esprit critique face à une programmation davantage diversifiée ${ }^{42}$.

La question de la circulation des discours amène celle de la production et du financement. Le fonctionnement du CIRTEF est alors révélateur de la prise en compte des contraintes à la formation puis à la production qui existent sur le continent africain. Outre les 
opportunités de diffusion des œuvres sélectionnées, le CIRTEF propose également des ateliers de formation, gère sur le continent des centres de post-production équipés (à Cotonou, Yaoundé, Niamey et Maurice), et diffuse enfin des appels d'offres afin de financer des projets.

Si les réalisateurs occidentaux témoignent de fortes difficultés de financement pour des projets qui ont finalement une portée toute personnelle pour eux ${ }^{43}$, les deux documentaires Vivre de sable et Le cri de la mer, n'ont par exemple pu voir le jour qu'à travers la réponse à un appel d'offre organisé par le CIRTEF dans le cadre d'une série intitulée «Femmes battantes ». Ces deux projets sélectionnés ont ainsi été pris en charge dans leur intégralité, de l'écriture du scénario en résidence, au financement du tournage, au montage et post-production, également en résidence, jusqu'à la diffusion auprès de tout le réseau de diffusion du CIRTEF (TV5 Monde, RTBF, et télévisions publiques africaines). De la même façon, le documentaire Un ami est parti est un film de fin de formation, réalisé par Delphe Kifouani (2008) dans le cadre de ses études à Saint-Louis ${ }^{44}$ et financé par les partenaires de la formation (ministère de la Culture français et la Communauté francophone de Belgique $)^{45}$. Le cas du court-métrage de Mahamat-Saleh Haroun (2008) est différent, le réalisateur tchadien bénéficiant d'une reconnaissance internationale grâce en particulier au festival de Cannes, et son court-métrage a été financé dans le cadre du «Digital Project » du Festival international de films de Jeonju qui a eu lieu en Corée en 2008.

Ces exemples de réseaux de production et de coproduction illustrent à quel point la circulation des discours africains sur les migrations subsahariennes reste extrêmement conditionnée par les sources de financement largement situées en Occident. S'ils laissent une certaine autonomie aux réalisateurs, ces réseaux, de par leur manque de diversitée ${ }^{46}$, conduisent également à un certain formatage.

En outre, alors que les différentes mises en scène observées mettent en avant des trajectoires et des expériences individuelles, les messages politiques portés par ces œuvres de création restent relativement conformistes et n'opèrent pas un renversement effectif des stéréotypes liés aux questions migratoires, toujours reliées aux questions de la pauvreté et du manque de moyens. Sur la forme, le CIRTEF œuvre en particulier à la mise aux normes des productions audiovisuelles africaines afin qu'elles puissent être diffusées sur un réseau international ${ }^{47}$.

Mais le formatage des productions ne s'arrête parfois pas à la question des normes techniques et certains réalisateurs, comme Aïcha Thiam, expriment clairement les contraintes artistiques qui les limitent dans le cadre de projets répondant à des appels d'offres comme cela a été le cas pour Le cri de la mer.

«En fait, quand tu travailles avec le CIRTEF, tu as le problème parce que tu n'as pas la liberté artistique, mais au moins tu as la chance que ton projet voit le jour ! [...] Je suis contente de ce film parce que, il est venu à un moment où on était en plein discours sur l'immigration, où y'avait presque dix mille morts sur les côtes espagnoles, où les jeunes étaient prêts à tout pour partir et sur ce plan là, je suis contente d'avoir fait ce film. Après artistiquement, c'est pas l'un de mes films que je préfère. De ma filmographie. Parce qu'il y avait quand même une ligne éditoriale dont il fallait pas sortir. Un synopsis. C'est une série harmonisée. Il y avait huit films qui avaient été réalisés l'année passée, et nous on venait après. Et il fallait pas sortir de cette façon de faire le film: rencontrer un personnage, faire des interviews, filmer un peu son quotidien ${ }^{48}$. 
61 La réalisatrice met bien ici en valeur l'ambiguïté de la collaboration avec des organismes comme le CIRTEF, facilitateur et accélérateur de production, mais également entrave à la créativité. Pour une chaîne de télévision comme TV5 Monde, tournée vers la communauté francophone internationale et qui affirme son identité à travers les notions de métissage, d'interculturalité, et d'échanges, ces récits des migrations qui se distinguent des discours journalistiques prennent une valeur particulière, mettant en avant la polyphonie défendue par la chaîne.

Dans le dernier plan stratégique de la chaîne, son ancienne directrice annonçait son intention d' " acquérir et diffuser plus d'émissions et de films africains », en particulier par l'intermédiaire du CIRTEF «auquel une partie des recettes publicitaires de TV5 est reversée pour contribuer au financement des productions audiovisuelles africaines ». Denise Epoté, directrice de TV5 Afrique, souligne également le rôle de sa chaîne pour le continent : « Les émissions de TV5 sont une tribune pour l'Afrique. Il est important qu'elle puisse s'adresser au reste du monde $»^{49}$. Parallèlement à cette préoccupation, TV5 Monde multiplie depuis quelques années en Afrique la mise en place des "Maisons TV5 Monde ", espaces de convivialité, équipés en informatique pour accéder aux services en ligne de la chaîne, mais surtout permettant à ses téléspectateurs de se rassembler devant un écran géant pour regarder ses programmes.

Derrière ces discours de communication et ces dispositifs se cachent une réalité certaine, celle de la difficulté pour les auteurs du continent de faire entendre leur point de vue : d'une part sur le continent africain où les structures de production et de diffusion sont encore très marginales, et d'autre part à l'international. Il est alors intéressant de constater que la cartographie des espaces de diffusion des œuvres que nous avons étudiées retrace la cartographie des festivals francophones et internationaux consacrés au continent africain, ces derniers constituant le réseau principal de diffusion de ces œuvres audiovisuelles.

TV5 Monde paraît alors se structurer de manière complexe autour d'un double mouvement de " décentrage/recentrage $»^{50}$, recentrage avec les objectifs de son actionnaire principal, l'Audiovisuel extérieur français, qui a pour mission de «développer l'influence de la France dans le monde avec un double objectif, celui de la Francophonie et de la Francophilie $»^{51}$, décentrage avec sa structure multilatérale et la volonté de se faire le porte-voix de la diversité culturelle et des échanges entre les cultures ${ }^{52}$.

$\mathrm{V}$

65 Si nous restons dans le contexte formaté du discours télévisuel, ces œuvres de création diffusées par TV5 Monde ont un rôle de contre-point du fait qu'elles tranchent avec les discours journalistiques. Les sensibilités particulières, les émotions individuelles et les parcours de vie qui sont véhiculés complètent d'une certaine manière les discours que l'on trouve dans les programmes d'information et de débats à travers leur statut de témoignage tout à fait particulier dans la programmation. La migration n'est plus présentée comme un phénomène, mais comme une expérience qui est donnée à voir aux téléspectateurs au travers des portraits singuliers. De nouvelles facettes apparaissent et la question migratoire devient parfois l'occasion d'interroger plus largement les sociétés africaines. "Quand les réalisateurs prennent le parti d'une écriture audiovisuelle médiatrice » (Gellereau $1998: 7)$, ils peuvent alors faire office de passeurs, à l'image des écrivains de l'immigration qui élaborent, d'après Christiane Albert (2005: 155), « des espaces de médiation entre plusieurs langues, plusieurs histoires, plusieurs imaginaires et 
peuvent de ce fait rendre compte du métissage des cultures dont l'immigration est une des manifestations les plus emblématiques ».

Si le rôle de contre-point est certain, la logique de programmation de la chaîne demande toutefois de nuancer son ampleur dans la mesure où TV5 Monde apparaît, non sans contradiction, comme une chaîne multilatérale soucieuse de mettre en avant des regards multiples, en particulier les points de vue africains, dont elle se présente comme la tribune. Ces récits médiatiques, issus du Nord et du Sud, ont émergé dans un contexte de production extrêmement réduit et trouvent ainsi une audience internationale qui leur permet d'exister.

L'analyse des films, qui mettent en scène d'autres réalités que les discours journalistiques, nous a permis de faire émerger en avant les enjeux de leur diffusion dans le contexte moderne de globalisation qu'Arjun Appaduraï (2005: 31) définit comme marqué par le flux des personnes et le flux des images. Dans ce contexte, que l'on peut aisément associer à celui des média-cultures, « le travail de l'imagination ouvre un espace de contestation dans lequel les individus et les groupes cherchent à annexer le monde global dans leurs propres pratiques de la modernité ».

\section{BIBLIOGRAPHIE}

\section{Filmographie}

CHRISTOPHE, F., 2003, Facteur Toubab, Mille et Un Films, TV Rennes, Rennes cité média, 63 min.

CHRISTOPHE, F., 2005, Vacances au Sénégal, Mille et Un Films, TV Rennes, Rennes cité média, 66 min., diffusé sur TV5 Monde FBS le 16/09/2008.

DIAKITE, R., 2007, Vivre de sable, CIRTEF, RTS, 26 min., diffusé sur TV5 Monde FBS le 05/05/2005.

HAROUn, M.-S., 2008, Expectations, Pilifilms, 29 min., diffusé sur TV5 Monde FBS le 04/03/2009.

KIFOUANI, D., 2008, Un ami est parti, GSARA, Université Gaston Berger de Saint-Louis, diffusé sur TV5 Monde FBS le 06/06/2009.

LION, J.-J., 2008, Aminata rencontre Aminata, Cityzen TV, Fag Prod, 52 min., diffusé sur TV5 Monde FBS le 09/05/2009.

LUKENZO, J., 2010, Les immigrés, Patou Films International, série de 84 épisodes de 26 min. répartis en deux saisons.

NICE, F., 2007, Atterrissage. Le message de Yaguine et Fodé, CIRTEF, RTBF, 13 min., diffusé sur TV5 Monde FBS le 03/06/2006.

THIAM, A., 2008, Le cri de la mer, CIRTEF, RTS, 26 min., diffusé sur TV5 Monde FBS le 18/04/2009. WOUASSI, F., 2003, Afrique Années 60, Mago prod, 8 min., diffusé sur TV5 Monde FBS le 15/02/2006. 


\section{NOTES}

1. Jean-Paul MARTHOz (2011) reprend ici la notion de "glocalization », développée par le sociologue américain Roland ROBERTSON (1992).

2. Nous distinguons ici les discours journalistiques des œuvres de création désignant les fictions ou les documentaires.

3. Le corpus d'étude sera présenté en détail dans la première partie de l'article.

4. Éric MACE et Éric MAIGRET (2005: 10) définissent les médiacultures comme "points d'intersection des phénomènes démocratiques contemporains de construction du sens et de la valeur ", nécessitant de "décloisonner l'étude sur les médias (traditionnellement réservée aux spécialistes de la communication) de celle sur les cultures (apanage des spécialistes de l'art, du cinéma, de la culture) et de celle sur les politiques de représentations (réservée aux penseurs du politique)».

5. La notion de genre audiovisuel nous permet de revenir sur la distinction qu'effectue Patrick CHARAUDEAU (1997: 85) quand il distingue le discours médiatique à visée informative des autres discours audiovisuels. Ce critère de la visée informative peut alors nous être utile afin de mieux comprendre ce que nous appelons les discours journalistiques (à visée informative) et les œuvres de création (dont les visées sont autres, ou dont la visée informative s'accompagne d'autres objectifs tout aussi importants).

6. La période de recherche est établie du $1^{\mathrm{er}}$ janvier 2006 (date de passage de TV5 à TV5 Monde) au 31 décembre 2010. La recherche s'est concentrée pour des raisons d'accessibilité sur le canal FBS (France-Belgique-Suisse) de la chaîne, le dépôt légal de l'INA n'enregistrant que ce canal. Dans le cadre de notre thèse, nous travaillons également sur la programmation de France 24, mais cette chaîne d'information en continu ne diffusant pas d'œuvres de création, nous n'utiliserons pas ce corpus dans cette analyse. Notre corpus global comptabilise donc respectivement 30 documents pour TV5 Monde et 79 pour France 24.

7. Nous prendrons ici la définition de l'indexation que Bruno BACHIMONT (LESPINASSE, HABERT \& B ACHIMONT 2000: 2) indique dans son analyse du cas précis du travail d'indexation à l'INA : " paraphrase structurée d'un document dans une langue naturelle ou contrôlée rendant le document exploitable en vue d'un usage donné ».

8. Ibid.

9. Un thésaurus hiérarchise l'ensemble des mots-clés d'une base de données.

10. Cette racine permettait d'accéder à l'ensemble de la famille comprenant ainsi l'ensemble des préfixes et des suffixes ou des dérivations qui peuvent l'accompagner.

11. Bruno васнімоnт (ibid.) prend l'exemple d'une "enquête de la bibliothèque municipale de Nanterre, en 1993, qui évalue à 30 \% les mots-clés communs à deux groupes d'indexeurs qui décrivent le même document, à l'aide du même thésaurus ».

12. La grille d'analyse nous a permis de faire pour chaque séquence l'inventaire des sons (les prises de parole, les commentaires, l'ajout de musique) et des images (les différents types de plan, les mouvements de caméra, le détail de ce qui était filmé). 
13. À l'heure de l'écriture de l'article, nous avons mené des entretiens avec six des huit réalisateurs: Françoise Nice, Jean-Jacques Lion, Aïcha Thiam, François Christophe, Ramatou Diakite et Delphe Kifouani.

14. Denise Époté, directrice de TV5 Afrique. Salin FRANCK, «TV5 Monde met le cap sur l'Afrique. Les nouvelles ambitions africaines de la chaîne internationale francophone ", Afrik.com, 15 juillet 2008, <http://www.afrik.com/article14807.html\#nh1>, consulté le 14 juillet 2012.

15. Depuis 2013, le capital de TV5 Monde est détenu à $49 \%$ par France Télévisions, à 12,58 \% par France Médias Monde, à 11,11 \% par la RTS, à 11,11 \% par la RTBF, à 11,11 \% par le Consortium Télévisions Canada Québec, à 3,29 \% par Arte France, à 1,74 \% par l'INA, et à $0,06 \%$ par les mandataires sociaux de la chaîne.

16. Dossier de presse TV5 Monde, 2010.

17. Présentation faite sur le site de la régie publicitaire de France Télévision, <http:// www.ftv-publicite.fr/spip.php?article439>, consulté le 14 juillet 2012.

18. Marie-Christine Saragosse est présidente directrice générale de l'AEF depuis le 7 octobre 2012, et c'est Yves Bigot qui l'a remplacée à la tête de TV5 Monde le 5 décembre 2012.

19. Salin FRANCK, «TV5 Monde met le cap sur l'Afrique. Les nouvelles ambitions africaines de la chaîne internationale francophone ", Afrik.com, 15 juillet 2008, < http:// www.afrik.com/article14807.html\#nh1>, consulté le 14 juillet 2012.

20. «Une terre n'est jamais aussi chère que lorsqu'on est loin d'elle. » Alain et Espérance sont français et noirs. Ils se télescopent, se toisent et s'écartent l'un de l'autre lorsqu'ils sont happés en plein cœur de Paris par une musique de Manu Dibango (<http:// www.africultures.com/php/index.php?nav=film\&no=667>).

21. "C'est l'histoire de Moussa, récemment revenu dans son village après un voyage infructueux dans le désert pour rejoindre la cohorte des candidats à l'émigration. Il se retrouve confronté aux hommes qui lui ont prêté l'argent du voyage» (<http:// www.pilifilms.fr/expectations/>).

22. «Yaguine et Fodé sont ces deux adolescents guinéens qui ont trouvé la mort dans des circonstances tragiques. Voulant fuir la misère, ils ont embarqué dans un avion à destination de la Belgique. Ils n'ont pas survécu au voyage. Partant de ce tragique fait divers européen, Denis Poumga et le théâtre musical possible ont monté la pièce atterrissage qui a fait l'ouverture du festival de théâtre international du Bénin » (<http:// www.clubcirtef.net>).

23. « Parmi tous mes amis, l'un d'eux, d'origine sénégalaise, m'est resté dans l'esprit. Il a été le premier à me parler de ma différence, du fait que je viens d'Afrique centrale. Il m'a fait connaître sa société, ses tabous. Aujourd'hui, je ne sais pas où il est. Je sais juste qu'un jour, il est parti sur une pirogue en direction de l'Europe» (<http:// www.africultures.com/,php/index.php?

nav=film\&no=7436\&texte_recherche=unamiestparti $>$ ).

24. "L'idée de la série, c'était de trouver des réalisatrices africaines qui font des documentaires sur des femmes africaines qui se battent dans leur communauté pour faire avancer les choses » (entretien avec Aïcha Thiam, le 20 juillet 2012).

25. «C'est la faim qui pousse les femmes de Zaraganda à choisir le chemin de l'exil. Chez elles, les champs arides ne donnent plus rien. Toutes celles qui en ont encore la force 
partent donc pour Niamey. Pendant de longs mois, de jour comme de nuit, elles parcourent le bitume et vendent leur travail au plus offrant » (<http://www.clubcirtef.net $>$ ).

26. "Le cri de la mer est le combat d'une mère, Yaye Bayam Diouf, qui a perdu son fils unique dans une pirogue en partance pour les îles Canaries. Aujourd'hui à Thiaroye-surMer, son quartier, sa vie est consacrée à la lutte contre l'émigration clandestine » (< http://www.africultures.com/php/index.php?

nav=evenement\&no=18728\&texte_recherche=le\%20cri\%20de\%20la\%20mer $>$ ).

27. "Après quatre années de clandestinité en Italie, Yelli, un ami sénégalais, a été régularisé. Il va enfin pouvoir voyager librement et revoir les siens. Toute la famille l'attend avec ses besoins immenses. Mais en Europe, Yelli a entrevu la possibilité de mener une vie différente » (<http://www.docnet.fr/product_info.php? products_id=54.com>).

28. « De son pays et de sa famille d'origine, Aminata ne connaît pas grand chose, alors elle a décidé de retourner à Finthiock, le petit village de Casamance où vivent aujourd'hui l'essentiel des membres de sa famille, notamment sa grand-mère dont elle porte le prénom: Aminata » (<http://www.africultures.com/php/index.php? nav=film\&no=7620\&texte_recherche=aminatarencontreaminata $>$ ).

29. Prenons pour exemple les nombreux reportages qui décrivent les dispositifs (humains, techniques, financiers, législatifs, etc.) mis en place par l'Union européenne pour lutter contre l'immigration clandestine. Ces reportages mettent la plupart du temps en scène une opposition entre les images des migrants qui arrivent sur les côtes méditerranéennes ou qui se font interpeller avant et les prises de parole des dirigeants européens. La question migratoire est alors principalement une question de chiffres (nombre de clandestins repoussés, nombre de personnes attendant au Maghreb de pouvoir traverser, budget alloué à Frontex, nombre d'hélicoptères qui patrouillent, etc.).

30. Entretien avec Delphe Kifouani, le 12 août 2012.

31. Dans notre corpus global, seuls deux reportages concernent des migrations Sud-Sud.

32. Nous évoquons ici la médiatisation de projets comme celui du CIGEM (Centre d'information et de gestion des migrations) au Mali, créé en 2008 par le Mali et l'Union européenne qui finance ce projet. Le CIGEM a officiellement pour mission d'améliorer la connaissance des phénomènes migratoires; d'accueillir et d'informer les migrants potentiels et des migrants de retour; de donner des informations juridiques et de sensibiliser sur les risques de la migration irrégulière; d'accompagner et valoriser les accords de co-développement entre le Mali et l'Union européenne.

33. Entretien avec Aïcha Thiam, le 20 juillet 2012.

34. Entretien avec Françoise Nice, le 20 juillet 2012.

35. Entretien avec Aïcha Thiam, le 20 juillet 2012.

36. Entretien avec Delphe Kifouani, le 12 août 2012.

37. Nous empruntons ici le terme de recentralisation à Koichi IWABUCHI (2008) qui pense la globalisation de la culture japonaise.

38. Nous retrouvons ici la déconstruction de la notion de local chère à Arjun APPADURAÏ (2005), qu'il préfère remplacer par « local imaginé ».

39. Deux autres étant programmées dans la collection "Sud côté court", émission diffusant une sélection de courts-métrages africains, également coproduite par le CIRTEF. 
40. Voir < http://www.tv5.org/TV5Site/enseigner-apprendre-francais/fiche-1005Reflets_Sud.htm>, consulté le 14 juillet 2012.

41. Vidéo pour les 20 ans en janvier 2012, <http://www.rtbf.be/video/v_reflets-sud? id=1556863>, consulté le 14 juillet 2012.

42. Entretien avec Mafarma Sanogo, le 22 août 2012. Lors de l'entretien, afin d'illustrer les différences de perception qui peuvent naître entre un regard interne et africain et un regard extérieur, Madame Sanogo prendra l'exemple de deux documentaires portant sur les enfants talibés au Sénégal.

43. Françoise Nice, la réalisatrice du documentaire sur Yaguine et Fodé a finalement monté son projet avec un budget de $3000 €$, sur des fonds qu'elle a en partie empruntés à sa famille, avant de vendre le produit fini au CIRTEF.

44. Master 2, "Réalisation documentaire de création", ouvert à l'Université Gaston Berger, à Saint-Louis au Sénégal, en coopération avec l'Université Stendhal de Grenoble en France. Notons alors qu'Aïcha Thiam, réalisatrice du Cri de la mer, est également issue de ce même master.

45. Delphe Kifouani faisant partie du groupe suivi par les Belges, le montage de son film s'est fait à l'occasion d'un déplacement organisé en Belgique.

46. Les acteurs concernés se concentrent finalement autour de quelques institutionsphares: l'UNESCO, l'Organisation internationale de la francophonie, certains ministères français et belges, Canal France International, le CIRTEF, TV5 et, à plus petite échelle, des organismes comme Africadoc qui travaillent sur le documentaire africain et sont en liaison avec les formations et structures existantes.

47. Le numérique est alors un enjeu essentiel et était d'ailleurs le thème du SEFOR 2012, rencontre annuelle organisée par le CIRTEF sur les enjeux de l'audiovisuel africain.

48. Entretien avec Aïcha Thiam, le 20 juillet 2012.

49. Stéphane BALLONG, "Quelle place pour l'Afrique dans la nouvelle stratégie de TV5 Monde? ", interview de Marie-Christine Saragosse, directrice générale de la chaîne, Afrik.com, 23 janvier 2009, <http://www.afrik.com/article16139.html>, consulté le 14 juillet 2012.

50. K. IWABUCHI (2008: 46) a choisi de traduire par "décentralisation/centralisation", mais nous préférons éviter la confusion avec la terminologie propre à l'aménagement du territoire en utilisant plutôt le duo décentrage/recentrage.

51. Voir < http://www.aefmonde.com/page/presentation-mission.html>, consulté le 14 juillet 2012.

52. Dossier de presse TV5 Monde, 2010.

\section{RÉSUMÉS}

La comparaison des œuvres de création qui portent sur les migrations subsahariennes avec les discours journalistiques au sein de la programmation de TV5 Monde permet de montrer le rôle de 
contre-point que possèdent les œuvres vis-à-vis du reste de la programmation. Les films permettent la rencontre avec des migrants et leurs proches, au travers de leurs parcours, de leurs émotions, de leurs motivations. Les portraits permettent d'accéder à des expériences plutôt qu'à un phénomène, et ces œuvres mettent alors en lumière tout l'enjeu de la diffusion de points de vue recentrés dans un contexte global et média-culturel.

African Migration in Creation Films on TV5 Monde

Comparison between creation films that focus on African migrations and journalistic discourses in the programming of TV5 Monde helps to show the role of counterparty owned works by the rest of the programming. Films allow encounters with migrants and their families, through their journey, their emotions, their motivations. Portraits provide access to experiences rather than a phenomenon, and then put these works highlight the challenge of disseminating views refocused in a global media and cultural context.

\section{INDEX}

Mots-clés : médiations, migrations subsahariennes, télévisions internationales, œuvres de création

Keywords : Mediations, African Migration, International TV Channels, TV5 Monde, Creation Films

\section{AUTEUR}

\section{NATHALIE NEGREL}

Laboratoire d'études et de recherches appliquées en sciences sociales (LERASS), Université Toulouse II-Le Mirail, Toulouse. 